\title{
Review \\ Host microenvironment in breast cancer development Extracellular matrix-stromal cell contribution to neoplastic phenotype of epithelial cells in the breast
}

\author{
Malathy PV Shekhar ${ }^{1,2}$, Robert Pauley ${ }^{1,3}$ and Gloria Heppner ${ }^{1,3}$ \\ ${ }^{1}$ Breast Cancer Program, Karmanos Cancer Institute, Wayne State University School of Medicine, Detroit, Michigan, USA \\ ${ }^{2}$ Department of Pathology, Wayne State University School of Medicine, Detroit, Michigan, USA \\ ${ }^{3}$ Department of Internal Medicine, Wayne State University School of Medicine, Detroit, Michigan, USA \\ Corresponding author: Malathy PV Shekhar (e-mail: shekharm@karmanos.org)
}

Published: 20 February 2003

Breast Cancer Res 2003, 5:130-135 (DOI 10.1186/bcr580)

(c) 2003 BioMed Central Ltd (Print ISSN 1465-5411; Online ISSN 1465-542X)

\begin{abstract}
Epithelial-mesenchymal interactions play an important role both in normal mammary gland development and during neoplastic transformation. Perturbations in the production, deposition and degradation of the extracellular matrix occurring during neoplastic transformation and progression have been implicated to arise from alterations in the stromal response. These changes in the stroma exhibit a dominant regulatory role, via microenvironmental epigenetic effectors, to contribute to the development of the tumorigenic epithelial phenotype. The role of stromally derived microenvironmental epigenetic effectors in modulating epithelial growth, hormonal response, morphogenesis and epithelial plasticity is discussed.
\end{abstract}

Keywords: basement membrane, estrogen, fibroblast, mammary epithelial cell, three-dimensional organization

\section{Introduction}

Both breast cancers and hyperplastic lesions of proliferative breast disease originate from epithelial cells of the terminal duct lobular unit [1]. Although cancer development proceeds through a multistep process in which epithelial cells undergo transformation as a result of multiple hits (genetic model), it is becoming increasingly evident that discrete genetic alterations in epithelial cells alone cannot explain multistep carcinogenesis whereby tumor cells are able to express diverse phenotypes during the complex phases of tumor development and progression. Several lines of evidence suggest that concomitant changes also occur in cells surrounding the epithelial neoplasms [2].

The epigenetic model posits that the host microenvironment exerts an initial inhibitory constraint on tumor growth that is followed by acceleration of tumor progression through complex cell-matrix interactions with the host stroma. Breast stroma accounts for more than $80 \%$ of the resting breast volume [3]. The stroma or the supportive platform for the epithelial layer is composed of fibroblasts, endothelial cells, smooth muscle cells, adipocytes, inflammatory cells, nerve cells and a macromolecular network of proteoglycans and glycoproteins collectively termed the extracellular matrix (ECM). Factors required for premalignant progression, growth of primary cancer as well as invasion and metastasis are all altered by stromal interactions. The present review will focus on the function of the stroma in regulation of epithelial growth, in regulation of morphogenesis and in regulation of epithelial plasticity.

A role for stromal cells in carcinogenesis has been suggested from studies of embryological development where instructive and permissive interactions, along with genetic factors, are required for programming and maintaining epithelial structure and function. The embryonic epithelial and instructive mesenchymal cells engage in a reciprocal molecular dialog that ensures proper organ development and function $[4,5]$. The permissive adult counterparts of these epithelial and stromal interactions are believed to 
provide the regulatory signals that maintain homeostasis. Malignant transformation of adult epithelial cells disrupts such homeostatic regulation, including the control of tissue architecture, adhesion, cell death and proliferation. The importance of epigenetic constraints and their impacts on the expression of the genetic machinery of the cancerous epithelium has been demonstrated by observations that tumors prefer to grow and metastasize from orthotopic sites rather than from ectopic sites $[6,7]$.

\section{Hormonal regulation by the stromal compartment}

The fibroblast is a major cell type of the stromal compartment and is intimately involved in orchestrating the stromal half of the dialog with the epithelium in maintaining tissue homeostasis $[8,9]$. Alterations in fibroblasts in the stroma adjacent to transformed epithelial cells have been documented in several tumor systems [10-12]. These include alterations in growth characteristics and in migratory potential, and altered expression of growth factors such as platelet-derived growth factor, insulin-like growth factor I and insulin-like growth factor II, transforming growth factor beta, hepatocyte growth factor and keratinocyte growth factor. However, the contribution of these stromal alterations to tumor development and growth has not been fully elucidated.

Results of tissue recombination studies utilizing epithelium and stroma from wild-type and estrogen receptor (ER) knockout mice have demonstrated that epithelial steroid receptors are neither necessary nor sufficient for hormonal regulation of epithelial proliferation. Instead, hormonal regulation of epithelial proliferation is a paracrine event mediated by ER-positive stromal cells [8]. Although these data signify the direct role that stroma plays in the control of hormone-mediated epithelial cell proliferation, these results are not completely applicable to humans as human breast stromal cells are devoid of ER [13]. This speciesspecific difference in stromal ER expression implies possible fundamental differences in regulation of epithelial growth and morphogenesis by the stroma between species. An emerging concept is that aromatase-mediated estrogen synthesis in stromal fibroblasts and/or tumor epithelial cells acts in a paracrine manner or an autocrine manner, respectively, to influence breast tumor growth [14]. Thus, in the early stages of breast cancer, a probable role for stromal fibroblasts is the local production of estrogen so that a paracrine mechanism dependent on estrogen/ER levels can drive epithelial expansion.

With neoplastic progression, this requirement for an external supply of estrogen may be replaced or additionally satisfied by production of estrogen by the cells themselves (autocrine action). Although the source of estrogen may be different in mice versus humans, an important implication is that these tumors may initially respond to endocrine perturbations as they are either estrogen dependent or estrogen responsive. In the case of estrogen-independent breast tumors, further growth of cancer cells may be achieved by autocrine action of autostimulatory growth factors that act either constitutively or under the positive control of estrogen. It is not clear, however, how hormonal regulation of growth factor networks is altered during tumor progression. Can changes in the regulation of stromal versus epithelial compartments by hormones have a role in the emergence of estrogen-independent epithelial growth?

Much work is clearly needed to clarify and define the hormonal integration and control of growth factor networks in vivo. It is apparent, however, that hormones can target both the epithelium and the stroma, and that a complex interplay exists among hormones and growth factors targeting these compartments. Variations in hormonal sensitivity resulting from receptor loss, alterations in estrogen synthesis that lead to differences in local estrogen levels, an altered ECM or altered stromal-epithelial interactions can thus lead to altered regulation of signaling mechanisms, and hence to disruption of normal regulation of proliferation and differentiation by growth factors [15]. Irregularities in the constituents of the stromal-epithelial milieu or aberrations in their interactions can induce genomic instability, can enhance tumor cell proliferation, and can drive both metastatic spread and progression to a hormone-refractory state. The inductive stroma may thus be more than a source of soluble growth factors and/or ECM to breast epithelial cells. The stroma may serve as a driving force to promote tumor progression by providing a platform to generate tumor cell heterogeneity [16-18].

\section{The ECM and function}

The ECM acts in several ways to modulate cell morphology and proliferation, acting as a substrate to which cells can adhere and as a reservoir of growth factors. The ECM is connected to the nucleus by a network of protein molecules that include transmembrane adhesion proteins, the cytoskeleton and the nuclear matrix. The matrix networks are different between normal and tumor cells, and modification of the ECM induces alterations in the composition of the nuclear matrix [19].

Studies utilizing transgenic mice have shown that the development of experimental mammary tumors is governed by a common epigenetic process; the progressive disruption of the glandular microenvironment [20]. Progressive and cumulative architectural changes in the transgenic glands often mimic those occurring during clinical breast cancer progression. These include multilayering of the epithelium in premalignant hyperplastic lesions, a more rare intraepithelial cellular disorganization that often leads to obliteration of the central lumen in nonmalignant carcinomas in situ, and an even more infrequent 
migration of epithelial-derived tumor cells into the surrounding stromal microenvironment in invasive carcinomas [21]. This increasing rarity of each architectural transformation suggests that microenvironmental suppression becomes more stringent as tumor progression proceeds.

A major epigenetic modulator of this suppression is the ECM [20], whose components are critical for enabling cells to determine their orientation and morphology. Laminin-mediated integrin signaling has been described to play an important role in apical/basal polarization of epithelial cells, in formation of a central lumen and in expression of a differentiation-specific $\beta$-casein gene product [22-24]. Perturbations in the production, deposition and degradation of the ECM occurring during neoplastic transformation and progression have been implicated to arise from alterations in the stromal response [25].

\section{Stromal dominance of neoplastic phenotypes}

Observations utilizing two-dimensional cell cultures in vitro have been critical in elucidating the genetic programs and signaling pathways that mediate the proliferation and hormonal response of malignant epithelial cells in breast cancers. However, cancer development is vastly more complex than simple autocrine dysregulation of epithelium. Cooperative paracrine interactions among diverse cell types in organized tissue structures are imperative for the determination of epithelial differentiation, proliferation and morphology. Understanding of the role of such interactions in normal human breast function and in breast cancers has been greatly facilitated by recent improvements in in vitro culture systems that recapitulate differentiated and transformed epithelial cell phenotypes.

Using a three-dimensional culture assay, developed with a reconstituted basement membrane, Weaver and colleagues $[26,27]$ demonstrated that the malignant phenotype of human breast cancer cells could be reversed by correcting ECM-integrin signaling. Various changes in integrin expression have been noted in breast carcinomas, although there are conflicting data with regard to the specific integrin subunits that are affected [28]. $\alpha_{6} \beta_{4}$-integrin signaling normally acts to initiate apical/basal polarity in response to contact with laminin in the basement membrane [29]. When cells become transformed, however, chronic activation of $\beta_{1}$-integrin signaling is seen at the expense of $\alpha_{6} \beta_{4}$-integrin, and cells no longer respond to epigenetic constraints imposed by the ECM [29]. Proliferation is controlled, morphogenesis is restored and tumorigenesis is dramatically reduced when this integrin switching is reversed, despite the fact that genetic abnormalities acquired by tumor cells during progression persist $[27,29]$. These data suggest that appropriate integrin signaling is one of the microenvironmental epigenetic effec- constraints in the epithelium, to suppress the expression of the breast cancer phenotype.

Further evidence for the dominant role of microenvironmental epigenetic effectors and their ability to influence epithelial cell plasticity has come from previous studies. Clear differences in epithelial growth and morphogenesis were observed when premalignant breast cells were cocultured on reconstituted basement membrane with normal breast fibroblasts versus tumor-derived breast fibroblasts [30]. Breast epithelial cells and breast fibroblast cells cultured separately over agar, to prevent adherence to the surface, organize into compact cellular spheroids. Both epithelial and fibroblast spheroids lack organization, although fibroblast spheroids exhibit functional differentiation forming a meshwork of type IV collagen ( $R$ Pauley, S Santner, L Tait, unpublished observations, 2002). In cocultures there is commonly compartmentalization with central fibroblasts and outer epithelial cells. Again epithelial morphogenesis is not evident, but fibroblast-differentiated function is indicated by a type IV collagen meshwork.

In the context of the present review, functionality for aromatase-mediated estrogen synthesis in fibroblast spheroids or in three-dimensional cocultures with epithelial cells can be postulated based on regulated aromatase activity in breast fibroblast monolayer cultures [14]. Cocultures over a reconstituted basement membrane and over agar exhibit similarities for epithelial fibroblast compartmentalization. Fundamental differences occur, however, because only cocultures over a reconstituted basement membrane exhibit epithelial morphogenesis, growth regulation and differentiation. These differences indicate that two fundamental components, the reconstituted basement membrane and the fibroblast, together influence epithelial neoplastic phenotype. In addition, the tissue origin of breast fibroblasts influences the epithelial neoplastic phenotype. On a reconstituted basement membrane, coculture of premaligant breast cells with normal fibroblasts resulted in only weak induction of epithelial growth and morphogenesis, but similar cocultures with benign or tumor-derived fibroblasts resulted in induction of highly proliferative ductal-alveolar morphogenesis. Interestingly, besides inhibiting morphologic transformation of premalignant breast cells, reduction mammoplasty-derived fibroblasts were also found to have the ability to suppress estrogen responsiveness of premalignant breast cells. Coculture with tumor-derived fibroblasts not only favored inductive morphogenetic and mitogenetic effects on premalignant breast cells, but also supported their responsiveness to estrogen [30].

These data suggest that breast fibroblasts derived from normal or tumor tissues have the ability to override/accentuate the genetic constraints imposed by the epithelial 
cells. It is interesting to note that, despite the presence of a variety of ECM proteins and factors in the reconstituted basement membrane matrix, the differentiation effects induced by the stroma are elicited only when premalignant breast cells are cocultured with organ-specific fibroblasts. These findings indicate a specific requirement for ECM molecules that are assembled or laid down by the mesenchyme for modeling of mammary glandular architecture, and they underscore the specific demands that must be met for establishment of productive stromal-epithelial interactions both by contact-dependent and soluble factor-mediated mechanisms.

\section{Genetic basis for stromal contribution to neoplastic phenotype}

Normal mammary terminal ductal-lobular units (TDLUs) adjacent to invasive breast cancers have been demonstrated to contain similar loss of heterozygosity (LOH), whereas TDLUs distant from cancerous components lacked such molecular heterogeneity [31]. These findings suggest that molecular alterations that characterize invasive breast carcinomas either may occur very early during progression or, at least in part, may represent a 'field effect' predictive of sensitivity to carcinogenic insult. The contribution of the stroma to early events in carcinogenesis, in the absence of exogenously induced mutagenic events in mammary epithelial cells, was demonstrated by significant increases in tumor incidence and by growth of COMMA-D cells transplanted to cleared mammary glands in irradiated hosts as compared with unirradiated animals [32]. The radiation-induced effects on stromal microenvironment were local rather than systemic because the tumors arose only when COMMA-D cells were transplanted to the irradiated side of animals [32].

Further support for the concept that the stroma is not an innocent bystander during epithelial carcinogenesis, but is an integral player in maintenance of cellular and tissue architecture, and perhaps a component of the 'field', has come from recent studies that have shown the presence of concurrent and independent genetic alterations in stromal and epithelial cells of human breast carcinomas [33,34]. The LOH frequency in the stroma close to cancer was found to range from 10 to $66.5 \%$ for ductal carcinoma in situ and from 20 to $75 \%$ for intraductal carcinomas, whereas no $\mathrm{LOH}$ was revealed in epithelial or stromal components of breast tissues from women without any breast disease [33]. These intriguing findings not only support the concept of stromal-epithelial interactions in the development and progression of breast neoplasia, but also suggest that genetic alterations in the stroma may significantly contribute to neoplastic phenotypes.

It is possible that some of these alterations may function in a dominant fashion while others may require the cooperation of parallel or multiple complex alterations at other sites to facilitate progression [35]. A more recent study has reported the presence of a lower frequency of $\mathrm{LOH}$ in stromal cells as compared with their epithelial counterparts microdissected from invasive adenocarcinomas [36]. These data suggest that stromal genetic alterations are either not dominant or may exert an indirect effect on the adjacent epithelium, or that they only exert an effect in collaboration with others to influence the overall process of tumorigenesis.

Alternatively, the presence of few, but critical, alterations in the stroma may be sufficient to play a key role in the regulation of epithelial proliferation and morphogenesis as stromal cells play a critical role in the production, assembly and dissolution of the ECM [18,37]. Consequently, the presence of even relatively few, but critical, alterations in stromal cells might greatly influence epithelial growth, hormonal response, tumor invasion and/or dissemination through ECM remodeling [38-40]. If so, intratumor and intertumor heterogeneity of human breast cancer should be viewed not only as a result of phenotypic drifting arising from genomic instability, but also from the point of view of their impact in concert with the microenvironment and on the inherently dominant ability of the stroma to influence epithelial plasticity. The identification of critical stromally derived genes will be pivotal to the design of novel therapeutic strategies to selectively target the stromal microenvironment and to induce conversion of cancer cells to a benign or less aggressive state.

\section{Conclusions}

Breast neoplasia has traditionally focused on the tumorigenic transformation of breast epithelial cells resulting from the accumulation of genetic alterations in epithelial cells that influence fundamental processes including proliferation and organization. Nevertheless, concurrent structural, cellular, functional and genetic alterations occur in the surrounding stroma. These changes in the stroma alter the normal stroma's influence on the fundamental epithelial cell characteristics of growth, morphogenesis and plasticity to contribute to the development of the tumorigenic epithelial phenotype. For example, alterations to fibroblasts adjacent to the transformed epithelium involve proliferation, growth factor expression and even local estrogen production. These alterations of fibroblast function are demonstrated to exhibit a dominant regulatory role via microenvironmental epigenetic effectors on the phenotype of breast epithelial cells.

Although details of concurrent and independent genetic alterations in human breast epithelial and stromal cells remain to be resolved, there is now substantive experimental evidence that stromal cells and the ECM interact both directly and indirectly to influence the phenotype of transformed breast epithelial cells. This provides the opportunity to identify and use new therapies that antagonize the 


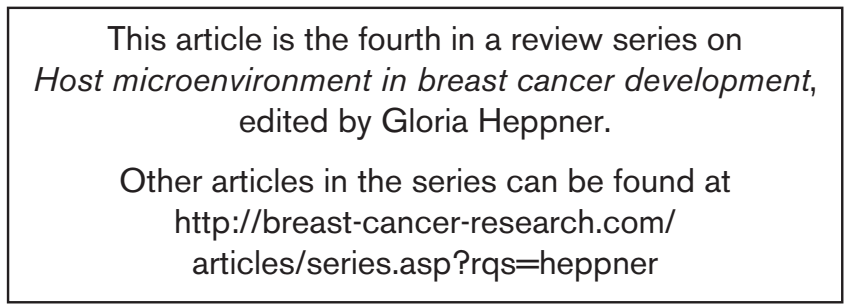

tumor-promoting influence of a tumor's stromal cells to reduce the tumorigenicity of transformed, and potentially invasive, breast epithelial cells.

\section{Competing interests}

None declared.

\section{Acknowledgements}

The research was supported by grants from the US Army Medical Research and Material Command (DAMD17-99-I-9443 and DAMD1702-I-0618 to PVMS, DAMD17-97-I-7171 to RJP) and from the National Institutes of Health (CA22453, Cancer Center Support Grant to the Karmanos Cancer Institute).

\section{References}

1. Heber D, Ashley J, Bagga D: Stromal-epithelial cell interactions in breast cancer. In Dietary Lipids, Hormones and Tumorigenesis. Edited by Kritchevsky HD. New York: Plenum Press; 1999:41-51.

2. Ronnov-Jessen L, Petersen OW, Bissell MJ: Cellular changes involved in conversion of normal to malignant breast - importance of the stromal reaction. Physiol Rev 1996, 76:69-125.

3. Drife JO: Breast development in puberty. In Endocrinology of the Breast: Basic and Clinical Aspects. Annals of the New York Academy of Sciences. Edited by Angeli A, Bradlow HL, Dogliotti L. New York: The New York Academy of Sciences; 1986:58-65.

4. Cunha GR, Alarid ET, Turner T, Donjaccour AA, Boutin EL, Foster $B A$ : Normal and abnormal development of the male urogenital tract: role of androgens, mesenchymal-epithelial interactions and growth factors. J Androl 1992, 13:465-475.

5. Kratochwil K: Tissue combinations and organ culture studies in the development of the embryonic mammary gland. In Developmental Biology: A Comprehensive Synthesis, vol 4. Edited by Gwatkin RBL. New York: Plenum Publishing; 1987: 315-334.

6. Gleave ME, Hsieh JT, von Eschenbach AC, Chung LWK: Prostate and bone fibroblasts induce human prostate cancer growth in vivo: implications for bidirectional tumor-stromal cell interaction in prostate carcinoma growth and metastasis. $J$ Urol 1992, 147:1151-1159.

7. Stephenson RA, Dinney CPN, Gohji Kordonez NG, Killion JJ, Fidler IJ: Metastatic model for human prostate cancer using orthotopic implantation in nude mice. J Nat/ Cancer Inst 1992, 84:951-957.

8. Cunha GR, Young P, Hom YK, Cooke PS, Taylor JA, Lubahn DB: Elucidation of a role for stromal steroid hormone receptors in mammary gland growth and development using tissue recombinants. J Mammary Gland Biol Neoplasia 1997, 2:393402.

9. Haslam SZ: Stromal-epithelial interactions in normal and neoplastic mammary gland. In Regulatory Mechanisms in Breast Cancer. Edited by Lippman M, Dickson R. Dordrecht: Kluwer Academic Publishers; 1991:401-420.

10. Chiquet-Ehrissmann R, Mackie EJ, Pearson CA, Sakakura T: Tenascin: an extracellular matrix protein involved in tissue interactions during fetal development and oncogenesis. Cell 1986, 47:131-139.

11. Singer C, Rasmussen A, Smith HS, Lippman ME, Lynch HT, Cullen KJ: Malignant breast epithelium selects for insulin-like growth factor II expression in breast stroma: evidence for paracrine function. Cancer Res 1995, 55:2448-2454.
12. Yee $\mathrm{D}$, Rosen $\mathrm{N}$, Favoni RE, Cullen $\mathrm{KJ}$ : The insulin-like growth factors, their receptors, and their binding proteins in human breast cancer. Cancer Treat Res 1991, 53:93-106.

13. Russo J, Ao X, Grill C, Russo IH: Pattern of distribution of cells positive for estrogen receptor $\alpha$ and progesterone receptor in relation to proliferating cells in the mammary gland. Breast Cancer Res Treat 1999, 53:21 7-227.

14. Pauley RJ, Santner SJ, Tait LR, Bright RK, Santen RJ: Regulated CYP19 aromatase transcription in breast stromal fibroblasts. J Clin Endocrinol Metab 2000, 85: 837-846.

15. Imagawa W, Pedchenko VK, Helber J, Zhang H: Hormone/ growth factor interactions mediating epithelial/stromal communication in mammary gland development and carcinogenesis. J Steroid Biochem Mol Biol 2002, 80:213-230.

16. Schor AM, Rushton G, Ferguson JE, Howell A, Redford J, Schor SL: Phenotypic heterogeneity in breast fibroblasts: functional anomaly in fibroblasts from histologically normal tissue adjacent to carcinoma. Int J Cancer 1994, 59:25-32.

17. Hasebe T, Sasaki S, Imoto S, Ochiai A. Highly proliferative fibroblasts forming fibrotic focus govern metastasis of invasive ductal carcinoma of the breast. Mod Pathol 2001, 14:325-337.

18. Wolf $C$, Rouyer N, Lutz $Y$, Adida C, Loriot $M$, Bellocq JP, Chambon $P$, Basset $P$ : Stromelysin 3 belongs to a subgroup of proteinases expressed in breast carcinoma fibroblastic cells and possibly implicated in tumor progression. Proc Natl Acad Sci USA 1993, 90:1843-1847.

19. Getzenberg RH, Pienta KJ, Huang EY, Murphy BC, Coffey DS: Modifications of the intermediate filament and nuclear matrix networks by the extracellular matrix. Biochem Biophys Res Commun 1991, 179:340-344.

20. Cardiff RD, Anver MR, Gusterson BA, Hennighausen L, Jensen RA, Merino MJ, Rehm S, Russo J, Tavassoli FA, Wakefield LM, Ward JM, Green JE: The mammary pathology of genetically engineered mice: the consensus report and recommendations from the Annapolis meeting. Oncogene 2000, 19:966-967.

21. Roskelley CD, Bissell MJ: The dominance of the microenvironment in breast and ovarian cancer. Semin Cancer Biol 2002, 12:97-104.

22. Roskelley CD, Desprez PY, Bissell MJ: Extracellular-dependent, tissue-specific gene expression requires both physical and biochemical signal transduction. Proc Natl Acad Sci USA 1994, 91:12378-12382.

23. Muschler J, Lochter A, Roskelley CD, Yurchenco P, Bissell MJ: Division of labour among the $\alpha_{6} \beta_{4}$ integrin, $\beta_{1}$ integrins, and an E3 laminin receptor to signal morphogenesis and $\beta$-casein expression in mammary epithelial cells. Mol Biol Cell 1999, 10:2817-2828.

24. Somasiri AM, Wu C, Ellchuk T, Turley S, Roskelley CD: Phosphoinositide $3-\mathrm{OH}$ kinase is required for adherens junctiondependent mammary epithelial cell spheroid formation. Differentiation 2000, 66:116-125.

25. Leygue E, Snell L, Dotzlaw H, Hole K, Hiller-Hitchcock T, Roughley $\mathrm{PJ}$, Watson $\mathrm{PH}$, Murphy LC: Expression of lumican in human breast carcinoma. Cancer Res 1998, 58:1348-1352.

26. Weaver VM, Howlett AR, Langton-Webster B, Petersen OW, Bissell MJ: The development of a functionally relevant cell culture model of progressive human breast cancer. Semin Cancer Biol 1995, 6:175-184.

27. Weaver VM, Petersen OW, Wang F, Larabell CA, Briand $P$, Damsky C, Bissell MJ: Reversion of the malignant phenotype of human breast cells in three-dimensional culture and in vivo by integrin blocking antibodies. J Cell Biol 1997, 137:231-242.

28. Hansen RK, Bissell MJ: Tissue architecture and breast cancer: the role of extracellular matrix and steroid hormones. Endocr Relat Cancer 2000, 7:95-113.

29. Nievers MG, Schaapveid RQ, Sonnenberg A: Biology and function of hemidesmosomes. Matrix Biol 1999, 18:5-17.

30. Shekhar PVM, Werdell J, Santner S, Pauley RJ, Tait, L: Breast stroma plays a dominant regulatory role in breast epithelial growth and differentiation: implications for tumor development and progression. Cancer Res 2001, 61:1320-1326.

31. Deng G, Lu Y, Zlotnikov G, Thor AD, Smith HS: Loss of heterozygosity in normal tissue adjacent to breast carcinomas. Science 1996, 274:2057-2059.

32. Barcellos-Hoff $\mathrm{MH}$, Ravani SA: Irradiated mammary gland stroma promotes the expression of tumorigenic potential by unirradiated epithelial cells. Cancer Res 2000, 60:1254-1260. 
33. Moinfar F, Man YG, Arnold L, Bratthauer GL, Ratschek M, Tavassoli $F A$ : Concurrent and independent genetic alterations in stromal and epithelial cells of mammary carcinoma: implications for tumorigenesis. Cancer Res 2000, 60:2562-2566.

34. Wernert N, Locherbach C, Wellmann A, Behrens P, Hugel A: Presence of genetic alterations in microdissected stroma of human colon and breast cancers. Anticancer Res 2001, 21:2259-2264.

35. Moinfar F, Man YG, Bratthauer GL, Ratschek M, Tavassoli FA: Genetic abnormalities in mammary ductal intraepithelial neoplasia-flat type ('clinging ductal carcinoma in situ'): a simulator of normal mammary epithelium. Cancer 2000, 88: 2072-2081.

36. Kurose K, Hoshaw-Woodard S, Adeyinka A, Lemeshow S, Watson PH, Eng C: Genetic model of multi-step breast carcinogenesis involving the epithelium and stroma: clues to tumour-microenvironment interactions. Hum Mol Genet 2001, 10:1907-1913

37. Noel A, Hajitou A, L'Hoir C, Maquoi E, Baramova E, Lewalle JM, Remacle A, Kebers F, Brown P, Calberg-Bacq CM, Foidart JM: Inhibition of stromal matrix metalloproteinases: effects on breast-tumor promotion by fibroblasts. Int J Cancer 1998, 76:267-273.

38. Picard O, Rolland Y, Poupon MF: Fibroblast-dependent tumorigenicity of cells in nude mice: implication for implantation of metastases. Cancer Res 1986, 46:3290-3294.

39. Grey AM, Schor AM, Rushton G, Ellis J, Schor SL: Purification of the migration stimulating factor produced by fetal and breast cancer patient fibroblasts. Proc Natl Acad Sci USA 1989, 86:2438-2442

40. Camps JL, Chang SM, Hsu TC, Freeman MR, Hong SJ, Zhau HE, von Eschenbach AC, Chung LW: Fibroblast-mediated acceleration of human epithelial tumor growth in vivo. Proc Natl Acad Sci USA 1990, 87:75-79.

\section{Correspondence}

Malathy PV Shekhar, Breast Cancer Program, Karmanos Cancer Institute, 110 East Warren Avenue, Detroit, Ml 48201, USA. Tel: +1 313 833 0715, ext 2326/2259; fax: +1 313831 7518; e-mail: shekharm@karmanos.org 